\title{
Distance makes the difference in thermography for ecological studies
}

\author{
E. Faye ${ }^{\mathrm{a}, \mathrm{b}, \mathrm{c}, *}$, O. Dangles ${ }^{\mathrm{a}}, \mathrm{S}$. Pincebourde ${ }^{\mathrm{d}, * * *}$ \\ a UMR EGCE, IRD-247 CNRS-UP Sud-9191, 91198 Gif-sur-Yvette cedex, France \\ ${ }^{\mathrm{b}}$ Sorbonne Universités, UPMC Univ Paris 06, IFD, 4 Place Jussieu, 75252 Paris cedex 05, France \\ ${ }^{\text {c }}$ Pontifica Universidad Católica del Ecuador, Facultad de Ciencias Exactas y Naturales, Quito, Ecuador \\ ${ }^{\mathrm{d}}$ Institut de Recherche sur la Biologie de l'Insecte, UMR 7261, CNRS-Université François-Rabelais de Tours, 37200 Tours, France
}

\section{A R T I C L E I N F O}

\section{Article history:}

Received 26 July 2015

Received in revised form

27 November 2015

Accepted 30 November 2015

Available online 17 December 2015

Keywords:

Thermography

Thermal bias

Distance

Microclimate

Leaf temperature

\begin{abstract}
A B S T R A C T
Surface temperature drives many ecological processes and infrared thermography is widely used by ecologists to measure the thermal heterogeneity of different species' habitats. However, the potential bias in temperature readings caused by distance between the surface to be measured and the camera is still poorly acknowledged. We examined the effect of distance from 0.3 to $80 \mathrm{~m}$ on a variety of thermal metrics (mean temperature, standard deviation, patch richness and aggregation) under various weather conditions and for different structural complexity of the studied surface types (various surfaces with vegetation). We found that distance is a key modifier of the temperature measured by a thermal infrared camera. A non-linear relationship between distance and mean temperature, standard deviation and patch richness led to a rapid under-estimation of the thermal metrics within the first $20 \mathrm{~m}$ and then only a slight decrease between 20 and $80 \mathrm{~m}$ from the object. Solar radiation also enhanced the bias with increasing distance. Therefore, surface temperatures were under-estimated as distance increased and thermal mosaics were homogenized at long distances with a much stronger bias in the warmer than the colder parts of the distributions. The under-estimation of thermal metrics due to distance was explained by atmospheric composition and the pixel size effect. The structural complexity of the surface had little effect on the surface temperature bias. Finally, we provide general guidelines for ecologists to minimize inaccuracies caused by distance from the studied surface in thermography.
\end{abstract}

(c) 2015 Published by Elsevier Ltd.

\section{Introduction}

Surface temperature drives many physical, chemical, biological and ecological processes and is among the most influent factors for life across all biomes including marine, terrestrial and freshwater ecosystems (Oke, 1987; Kingsolver, 2009). Several methodologies have been developed to measure surface temperature. Among them, infrared thermography is the only non-invasive method that provides a continuous capture of surface temperature, and major developments over the past decade significantly improved our understanding of temperature-related patterns in ecological sciences (Quattrochi and Luvall, 1999; Cilulko et al., 2013; Lathlean and Seuront, 2014). Originally, infrared thermography was developed mainly for industrial, medical and military applications (Vollmer and Möllmann, 2010). It was first used for

\footnotetext{
* Corresponding author at: UMR EGCE, IRD-247 CNRS-UP Sud-9191, 91198 Gifsur-Yvette cedex, France.

** Corresponding author.

E-mail addresses: ehfaye@gmail.com (E. Faye), sylvain.pincebourde@univ-tours.fr (S. Pincebourde).
}

ecological research in the late sixties (e.g. studies on seal thermoregulation, Ørtisland 1968, and on white-tailed deer detection, Croon et al. 1968). Over the last four decades, infrared thermography has been increasingly used in various fields of biology including thermal physiology (Hill et al., 1980; Pincebourde et al., 2012; Woods, 2013; McCafferty et al., 2013), marine ecology (Lathlean and Seuront, 2014), plant sciences (Jones et al., 2002, Jones, 2013; Pincebourde and Woods, 2012; Caillon et al., 2014), agronomy (Jackson et al., 1981; Inagaki and Nachit, 2008; Meron et al., 2010; Bellvert et al., 2013), and landscape ecology (Scherrer and Koerner, 2010; Tonolla et al., 2010; Faye et al., 2015).

Infrared thermography is an imaging method that records infrared waves emitted by an object in the electromagnetic spectrum after the visible range of light - from 7.5 to $14 \mu \mathrm{m}$ - as a result of molecular motion (Vollmer and Möllmann, 2010). Radiation readings are then converted into surface temperature by the Thermal Infra-Red (TIR) camera taking into account ambient conditions and object's emissivity. TIR images allow the study of surface temperature patterns over a broad range of spatial scales from sea and land surface satellite mapping (Kerr and Ostrovsky, 2003) to landscape (Scherrer and Koerner, 2010; Faye et al., 2015) 
and organism scales (Tattersall and Cadena, 2010; Pincebourde et al., 2013). Recent advances in thermal imaging technology increasingly lightweight and hand-held - and a reduction in the cost of thermal cameras have facilitated its uses and opened new areas of investigation in ecological sciences (Lathlean and Seuront 2014; Faye et al. 2015).

However, despite its increasing use, relatively few studies have addressed the potential pitfalls and limits of thermal imaging (Clark, 1976; Quattrochi and Luvall, 1999; Minkina and Dudzik, 2009; Cilulko et al., 2013; Lathlean and Seuront, 2014). Weather conditions (e.g. solar radiation and rainfall) are known to affect TIR outputs leading to misinterpretation of organism body temperatures. Also, emissivity of an object - i.e. the ability of an object to emit thermal radiation - and viewing angle between the camera and the object can affect surface temperature measurements (Clark, 1976). Last, the distance between the object and the TIR camera (i.e. shooting distance) is among the main factors supposed to impact temperature values in TIR images (Nienaber et al., 2010; Cilulko et al., 2013). Like any image, TIR images are composed of pixels, and the portion of object surface area included in a single pixel directly depends on shooting distance - with larger area included in each pixel as shooting distance increases. Then, when the surface is thermally heterogeneous, neighbouring surface patches of different temperature merge together with increasing distance. To our knowledge, however, the net effect of increasing shooting distance on temperature readings by TIR camera has never been quantified. At best, TIR images are acquired at equal distances from the study organism allowing accurate estimates of relative temperature differences between patches (Inagaki and Nachit, 2008; Tonolla et al. 2010; Caillon et al., 2014).

Here, we examined the effect of shooting distance (in the range of $0.3-80 \mathrm{~m}$ ) on TIR thermal metrics that are commonly used to quantify the spatial heterogeneity of object temperatures (e.g. mean temperature, standard deviation, patch richness and aggregation). The aims of this study were (1) to characterize the relationship between these thermal metrics and shooting distance, (2) to assess the effect of weather conditions (solar radiation) on this relationship, and (3) to test whether the structural complexity of the studied surface affected this relationship. We first shot the same object surface (a thermal test card corresponding to a regular mosaic of black and white patches) under various global solar radiation levels with two similar TIR cameras placed at different distances. We then shot three object surfaces with different structure under identical global solar radiation with the two TIR cameras placed at various distances. Object surfaces consisted in a thermal test card under constant environmental conditions in the laboratory, a green wall covered by a deciduous woody vine scene, and an oak-beech forest edge offering a more complex scene. Additionally, we performed a TIR close-up shooting $(0.3 \mathrm{~m})$ of the plant leaves to assess how actual leaf temperatures shaped the surface temperature distribution at each shooting distance and to compare the micro-scale thermal heterogeneity of leaves to overall surface heterogeneity. Generally, we expected that the distance between the thermal camera and the studied object would lead to errors in the surface temperature because of the pixel size effect. We also expected this bias to be more pronounced when the surface is heated by solar radiation. Finally, under similar abiotic conditions, structurally complex surfaces are supposed to deliver more thermal heterogeneity than simpler ones and we hypothesized that the temperature measurements of these complex surfaces would be more influenced by shooting distance.

\section{Materials and methods}

\subsection{The thermal infrared cameras}

TIR images were acquired using two similar TIR cameras recording long-wave infrared radiation emitted by objects in the spectral range from 7.5 to $14 \mu \mathrm{m}$. They were equipped with uncooled micro-bolometer sensors and converted infrared radiation readings into temperatures within the -20 to $120^{\circ} \mathrm{C}$ calibration range. TIR images were processed assuming an emissivity of 1 for every surface because our interest was to quantify the discrepancies in spatial thermal heterogeneity between TIR images of the same surface taken at different distances - i.e. comparing relative values instead of measuring actual temperature values (Clark, 1976; Rubio et al., 1997). Therefore, surface temperature refers to the brightness surface temperature in this work (Norman and Becker, 1995). The surfaces we studied were almost entirely composed by vegetation, and mostly by leaf tissues. Emissivity of temperate tree leaves ranges between 0.95 and 0.98 (Gates 1980). A change in emissivity within this small range causes very small change in temperature readings. We are therefore confident that potential emissivity variations within the scenes did not cause the bias we observed. The first TIR camera (called fixed TIR camera, see below) was equipped with a $320 \times 240$ pixels micro-bolometer focal plane array (B335, FLIR Systems, Wilsonville, OR, USA). The second TIR camera (called mobile TIR camera, see below) was equipped with a $640 \times 480$ pixels micro-bolometer focal plane array (HR research 680, VarioCAMs, InfaTec GmbH, Dresden, Germany). For practical reasons, we did not use two identical TIR cameras. Therefore, we verified that the slight technical differences between the two cameras did not cause bias in surface temperature measurements (Online Resource 1). We shot studied surfaces simultaneously with both TIR cameras placed at each shooting distance from 2 to $80 \mathrm{~m}$ (see Online Resource 1 and below for details). We found no significant differences between the two TIR camera measurements on mean and standard deviation of temperatures and shooting distance did not significantly affect the small discrepancies between the two TIR cameras (Mann-Whitney-Wilcoxon Test, $P=21.92$ and 13.48 for mean and standard deviation respectively). Thus, the two TIR cameras yielded similar temperature readings.

\subsection{Experimental design}

\subsubsection{Thermal test card in different environments}

We studied a $1 \mathrm{~m}^{2}$ thermal test card, made of 400 black and 400 white tiles of $2.5 \mathrm{~cm}^{2}$ each, which delivered a well-characterized geometry and dimensions resulting in a predictable thermal pattern, with the black tiles reaching higher surface temperatures than the white ones when hit by radiation (Fig. 1). We placed the thermal test card vertically in three different environments that differed in term of abiotic parameters (exposure, temperature and global solar radiation). The first environment - the laboratory environment - was a $50 \mathrm{~m}$ long corridor without window in our laboratory (Institut de Recherche sur la Biologie de l'Insecte, Tours, France) wherein air temperature and humidity were maintained constant by an air-cooling system, thereby resulting in a homogeneous environment along the hall $\left(21.7^{\circ} \mathrm{C}\right.$ and $63 \%$ of humidity; Online Resource 2). Global radiation was generated using two $250 \mathrm{~W}$ metal halide bulbs (Sylvania Britelux HSI-T SX clear) positioned on the ground one metre in front of, and oriented toward, the thermal test card (A.1 and A.2 in Fig. 1). These lamps emitted both in the visible (37\% of total radiation) and in the near infrared range (63\% of total radiation) with a spectrum similar to solar radiation.

The second and third environments were outdoor, at the castle 


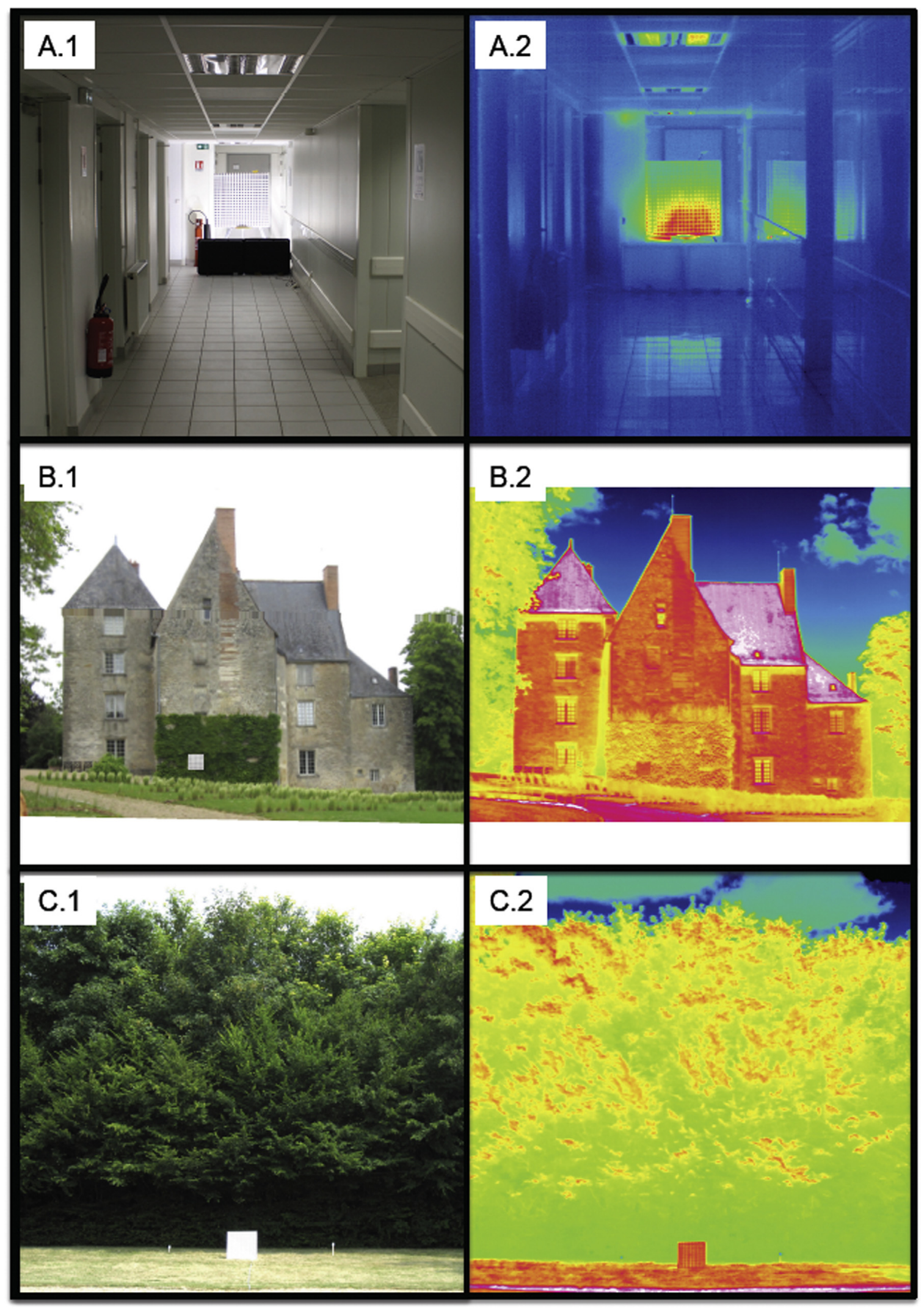

Fig. 1. RGB images (A.1, B.1, and C.1) and TIR images (A.2, B.2, and C.2) of the $1 \mathrm{~m}^{2}$ thermal test card placed in the three environments (laboratory A., green wall B. and wood edge C.) - Photos credits: Emile Faye (IRD) and Sylvain Pincebourde (CNRS).

named Château de Saché in the Loire Valley, France $\left(49^{\circ} 14^{\prime} 45^{\prime \prime} \mathrm{N}\right.$, $0^{\circ} 32^{\prime} 41^{\prime \prime} \mathrm{E}$, at a mean elevation of $77 \mathrm{~m}$ a.s.l.). In July 2013, when the study took place, mean daily temperature reached $20^{\circ} \mathrm{C}(27.7$ and $13.9{ }^{\circ} \mathrm{C}$ for mean maximum and minimum respectively) and photoperiod lasted almost 10 hours (Météo France, 2013). Thus, plants reached their fully-grown phenology with the highest vegetation density in canopies at that time (Koerner and Basler, 2010). We first placed the thermal test card in front of a South- 
exposed green wall of the castle - the green wall environment facing a flat area free of any obstacles (B.1 and B.2 in Fig. 1). Then, we positioned the thermal test card in front of a West-exposed wood edge in the court of the castle - the wood edge environment facing a flat area free of any obstacles (C.1 and C.2 in Fig. 1).

\subsubsection{TIR shots at increasing distances}

To test whether distance between the TIR camera and the object had an effect on the thermal metrics of surfaces, we used synchronised shots between the two TIR cameras placed at different distances in each environments (laboratory, green wall and wood edge). Synchronising shots allowed us to compare TIR images taken under exactly the same environmental conditions i.e. solar radiation and air temperature (Online Resource 1) - thus giving the effect of shooting distance directly. The fixed TIR camera was placed at a minimum distance from the surface so that it could capture a large extent: $2 \mathrm{~m}$ from the thermal test card in the laboratory, $3 \mathrm{~m}$ from the green wall and $10 \mathrm{~m}$ from the wood edge. The fixed TIR camera was considered to provide the most accurate surface temperatures, and the highest level of thermal heterogeneity, as it was placed at the shortest distance. The mobile TIR camera shot from distances to the fixed camera of 1,2 , and $7 \mathrm{~m}-\mathrm{i}$. e. distance at which $\Delta$ pixel size $\geq 0$ (Online Resource 1, Fig. 2) and up to 48,57 and $70 \mathrm{~m}$ in the laboratory, green wall and wood edge environments, respectively. One TIR shot was taken simultaneously with the two TIR cameras (less than 2 sec. differences between the two cameras, and each shot was repeated twice) at fourteen $\Delta$ distances (defined as the distance between the mobile and the fixed TIR cameras, see Online Resource 3 ) along a straight and perpendicular transect to the studied surface to avoid view angle effects on temperature readings (Clark, 1976). In total, we performed eight TIR shooting transects (two for the laboratory environment, three for the green wall environment and three for the wood edge environment) collecting up to $448 \mathrm{TIR}$ images under various abiotic conditions ( 8 TIR shooting transects $\times 14 \Delta$ distances $\times 2$ repetitions $\times 2$ TIR cameras). Each outdoor transect was performed between 11:23 and 13:49 to avoid important changes in solar radiation angles (Online Resource 2). At the end of each transect for the outdoor environments, we also took TIR images of leaf surfaces with the fixed TIR camera positioned at a distance of $0.3 \mathrm{~m}$ from the vegetation surface (Online Resource 4). Leaf surface temperature was measured for 15 shaded leaves and 15 leaves exposed to direct solar radiation. Initially, leaves were selected randomly and thereafter the same leaves
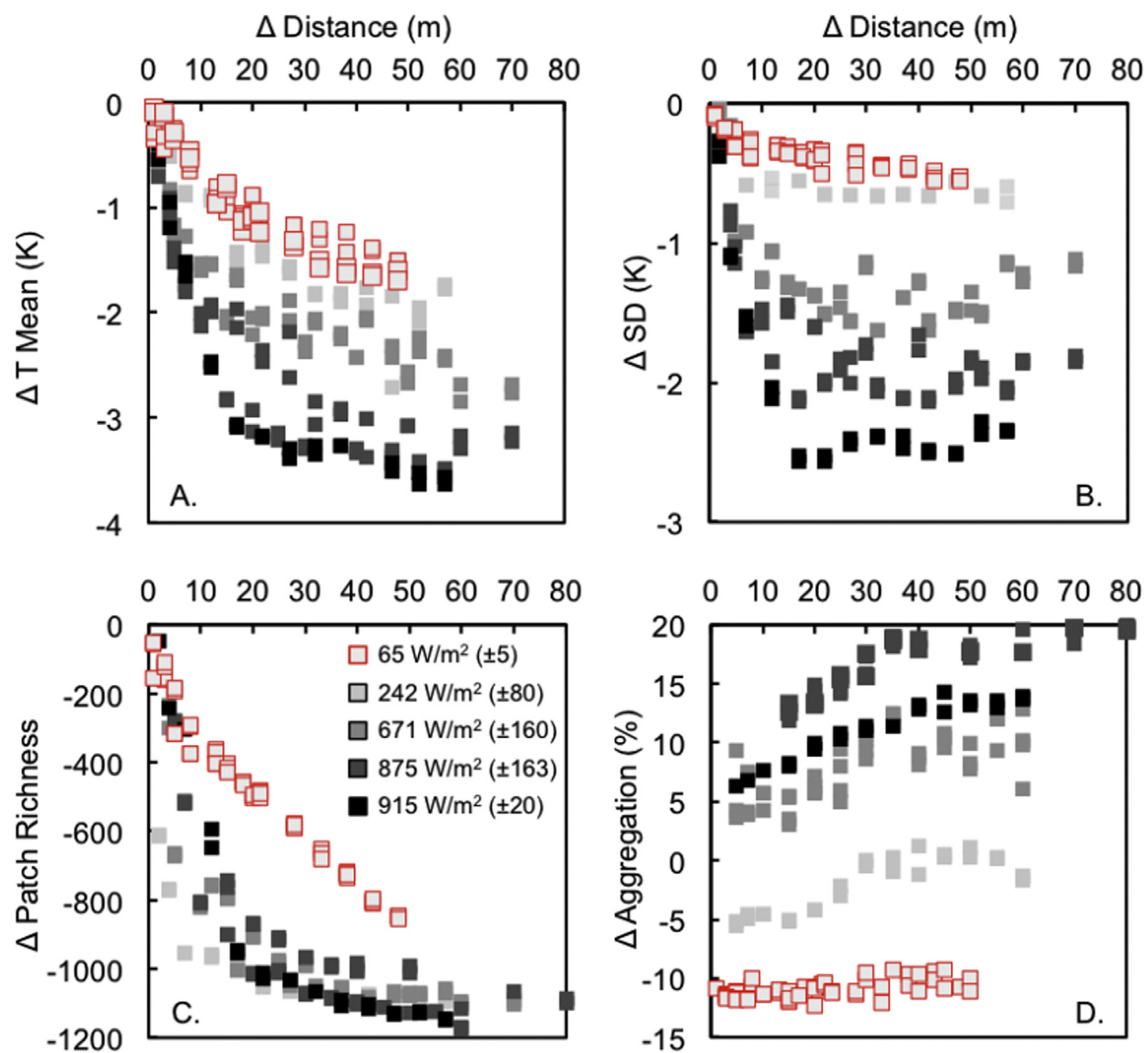

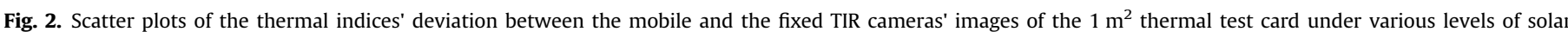

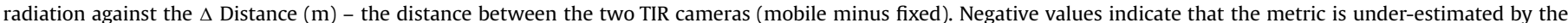

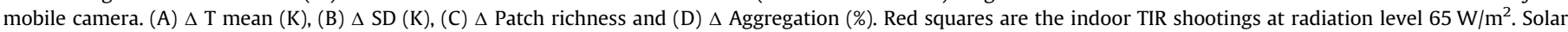

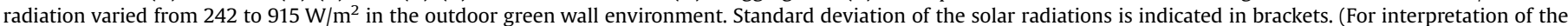
references to colour in this figure legend, the reader is referred to the web version of this article.) 

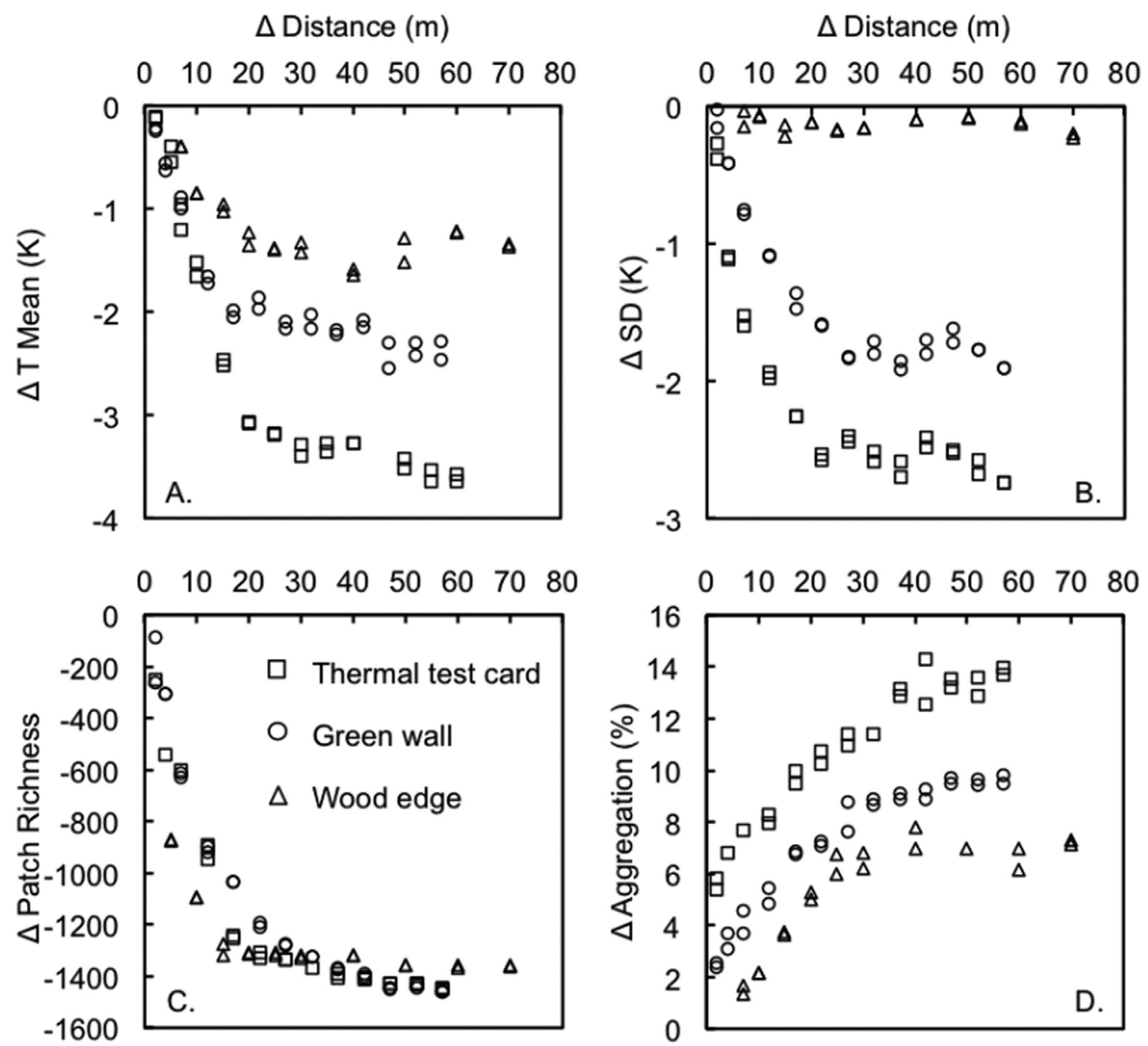

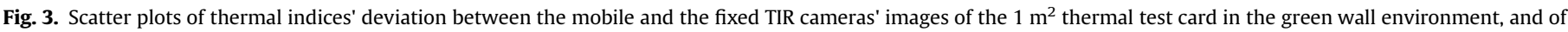

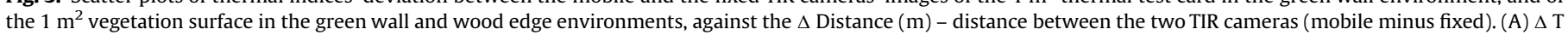
mean (K), (B) $\Delta$ SD (K), (C) $\Delta$ Patch richness, and (D) $\Delta$ Aggregation. Solar radiation was $890 \pm 133 \mathrm{~W} / \mathrm{m}^{2}$ for all points.

were measured during each session.

TIR cameras were switched on at least ten minutes before the beginning of each shooting to allow sensor stabilization. They were positioned on two professional tripods (MN 190X ProB, Manfrotto, Bassano Del Grappa, Italy) at $1.5 \mathrm{~m}$ above the ground to obtain a $90^{\circ}$ view angle to the surface (Clark, 1976). The angle of each camera relative to the surface was kept the same along each single transect. Simultaneously to each TIR image, we recorded global solar radiation (in $\mathrm{W} / \mathrm{m}^{2}$ ) using a datalogger equipped with a pyranometer sensor facing the sky vault (datalogger LI-200 and pyranometer LI-400, LI-COR, Lincoln, OR, USA).

\subsubsection{Differences among surfaces of different structural complexity}

To examine whether surface complexity modulated the effect of shooting distance on TIR outputs, we used surfaces differing in their structural complexity: (1) the thermal test card surface was the less structurally complex because of its well-defined twopatches composition in one plan; (2) the fully-grown grape ivy green wall (Parthenocissus tricuspidata) covering the south-exposed wall of the castle - background of the green wall environment - was a more structurally complex surface because of the various inclination angles of leaves that composed its almost two dimensional layout - the depth of the ivy cover did not exceed $20 \mathrm{~cm}$; (3) the third level of complexity consisted in a fully-grown wood edge composed of oak-trees (Quercus robur L.), beech-trees (Fagus sylvatica L.), and hornbeam-trees (Carpinus betulus L.) background of the wood edge environment -, which provided a highly complex surface composed of various patches in a threedimensional configuration with tens of metres in depth that increased the compositional heterogeneity. For each set of outdoor TIR images, we worked on two $1 \mathrm{~m}^{2}$ areas: the $1 \mathrm{~m}^{2}$ thermal test card (see above) and a $1 \mathrm{~m}^{2}$ area of vegetation located just beside the thermal test card in the green wall and wood edge environments (see TIR images in Online Resource 5).

\subsubsection{Surface temperature excess}

In order to determine the surface temperature excess - i.e. positive or negative deviation between pixel temperature values in the TIR images and ambient air temperature (Pincebourde and Woods, 2012), we measured ambient air temperatures using a set of temperature loggers (Hobo U23-001-Pro-V2, Onset Computer Corporation, Bourne, USA) placed within $5 \mathrm{~cm}$ behind the leaves and the thermal test card. The loggers were always shadowed and homogeneously distributed (20 loggers inside the green wall and the wood edge, and 10 more behind the thermal test card, see photographs in Online Resource 6). Temperatures were recorded every $10 \mathrm{~s}$ with an accuracy of $\pm 0.21 \mathrm{~K}$ and a resolution of $0.02 \mathrm{~K}$ at $25^{\circ} \mathrm{C}$. We standardized the TIR images using these air 
temperatures, which allowed us direct comparisons of leaf and surface temperature excesses in the two outdoor environments, regardless of their absolute temperature dissimilarities.

\subsection{Data analysis}

For each TIR image from the two TIR cameras, we marked the same $1 \mathrm{~m}^{2}$ area of the thermal test card and the same $1 \mathrm{~m}^{2}$ area of the vegetation surface (Online Resource 5). Pixel temperature values on these $1 \mathrm{~m}^{2}$ surfaces were extracted from raw images with ThermaCam Researcher software (FLIR Systems) and IRBIS 3 software (InfaTec $\mathrm{GmbH}$ ), from the fixed and the mobile TIR camera's images, respectively. We then calculated several thermal landscape indices from these pixel temperature matrices using FRAGSTATS (University of Massachusetts, Landscape Ecology Lab, Amherst, MA, USA): (1) mean temperature and standard deviation, providing a descriptive summary of patch metrics for the entire landscape, (2) patch richness, calculated as the number of patch types present in a landscape and describing its compositional make-up (McGarigal and Marks, 1994), (3) the aggregation index, often referred as landscape texture, which quantifies to what extent temperature pixels of the same value were spatially aggregated (He et al., 2000).

To analyse the effect of shooting distance on thermal metrics, we plotted the deviation in mean temperature $\left(\Delta \mathrm{T}_{\text {mean }}\right.$ in Kelvin), standard deviation ( $\Delta$ SD in Kelvin), patch richness ( $\Delta$ patch richness) and aggregation ( $\Delta$ aggregation in percentage) against the $\Delta$ Distance $(\mathrm{m})$ between the two TIR cameras (mobile camera minus fixed camera) for each surface. Those plots were represented for the various solar radiation levels in the three different environments (from 65 to $915 \mathrm{~W} / \mathrm{m}^{2}$, Fig. 2) and also for the three different surfaces - test card, green wall, wood edge - under similar and stable clear sky conditions (solar radiation of $890 \pm 133 \mathrm{~W} / \mathrm{m}^{2}$, Fig. 3).

We then searched for a general pattern in the change of thermal metrics with shooting distance by standardizing surface temperatures according to air temperatures (Online Resource 6). We plotted frequency curves of surface temperature excess of the thermal test card in the laboratory and in the green wall environment as function of shooting distance, and also of the entire green wall surface and of the entire wood edge surface under clear sky conditions (Fig. 4). For the outdoor environments, leaf surface temperature distributions were added to the plots to assess how actual leaf temperatures (i.e. leaf surface temperature distribution at high spatial resolution) shaped the surface temperature distribution from each shooting distance. For this analysis, we used the surface temperature excess matrices - the surface temperature distributions minus the mean ambient air temperature recorded by the temperature loggers behind leaves at the same time than TIR images (Online Resource 6). Densities were used to leave aside the effect of decreasing pixel number with increasing distance on the distribution curves, since the number of temperature pixels in the focused areas decreased with distance. As temperature frequency distributions were normal, they were fitted using Gaussian function in Table curve 2D (V5.01, Systat Software Inc., Chicago, Illinois, USA) as follows:

$F=a+b e^{\left[-0.5\left(\frac{T_{e x}-c}{d}\right)^{2}\right]}$

where $a, b, c$, and $d$ are fixed parameters, $F$ the frequency predicted and $T_{e x}$ the temperature excess in $\mathrm{K}$. The accuracy of the fits $\left(R^{2}\right.$ and standard deviation) of each density curve fitted is given in Online Resource 7. We performed an analysis of variance (ANOVA) with the R package 'stats' version 3.1.1 (R Development Core Team, 2015) to analyse the effects of shooting distance, radiation level and their interactive influences on surface temperature excess distributions.

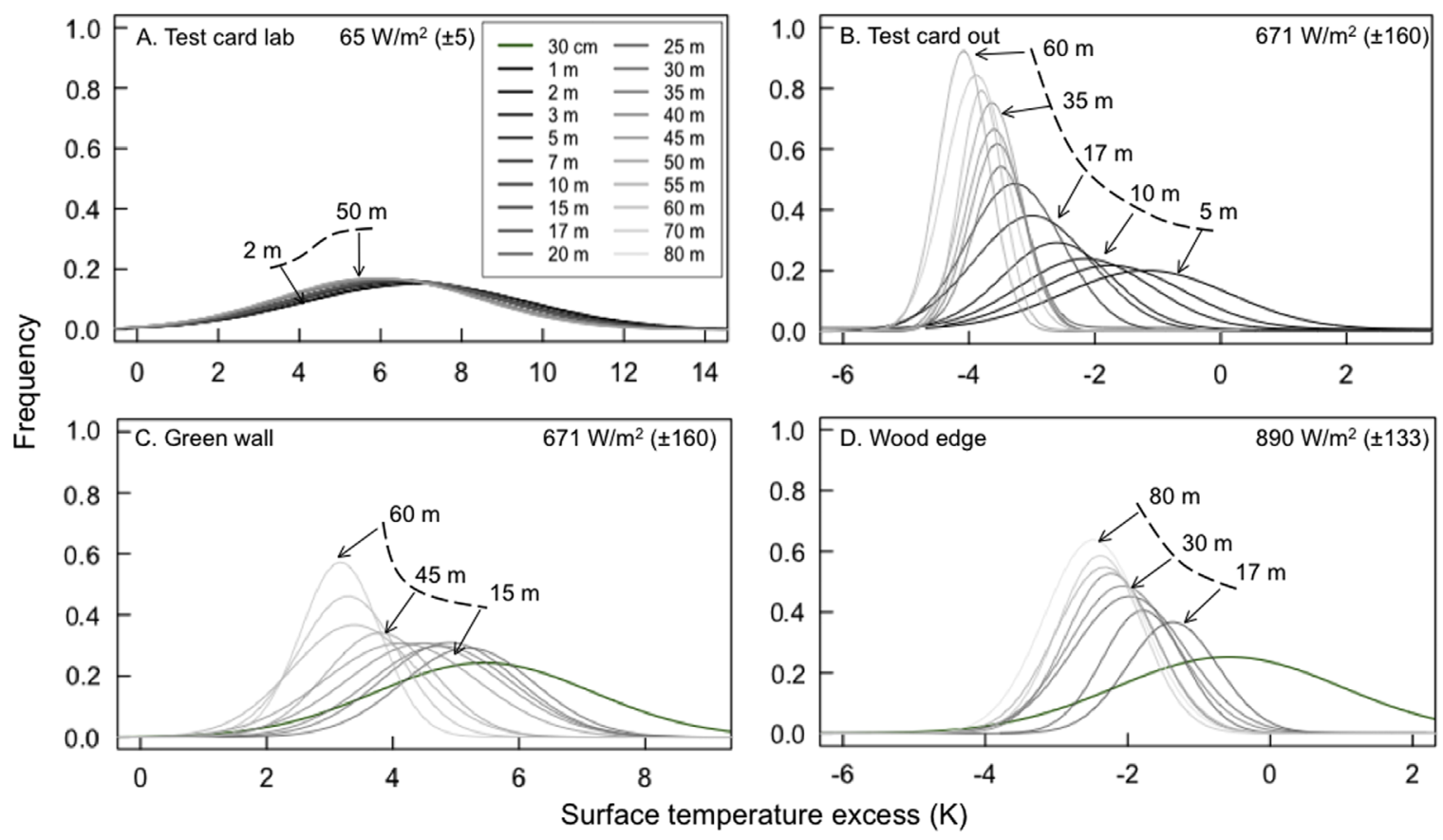

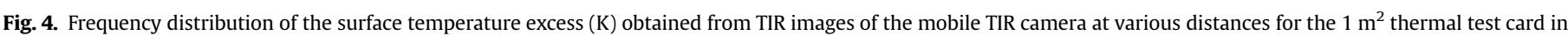

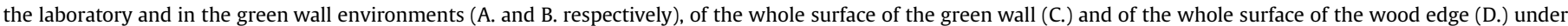

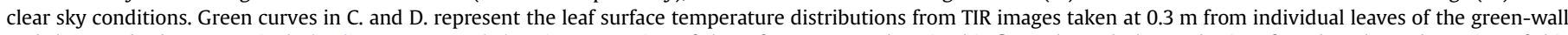

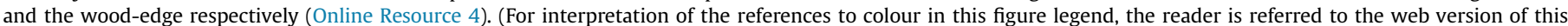
article.) 


\section{Results}

\subsection{Thermal test card in different environments}

Overall, the distance between the mobile and the fixed TIR cameras had a significant effect on all thermal metrics for the thermal test card ( $\Delta T_{\text {mean }}, \Delta \mathrm{SD}, \Delta$ Patch richness and $\Delta$ Aggregation; Fig. 2). Within the first $20 \mathrm{~m}$ separating the two TIR cameras, $\Delta T_{\text {mean }}, \Delta \mathrm{SD}$, and $\Delta$ Patch richness strongly decreased, from 0 to $-3.4 \mathrm{~K},-2.5 \mathrm{~K}$ and -1200 patches, respectively. At distances from 20 to $70 \mathrm{~m}$, this decrease was much less pronounced as it did not exceed $-1 \mathrm{~K},-0.8 \mathrm{~K},-400$ patches for $\Delta T_{\text {mean }}, \Delta \mathrm{SD}$, and $\Delta$ Patch richness respectively. $T_{\text {mean }}, \mathrm{SD}$, and Patch richness were therefore increasingly under-estimated as the distance between the two TIR cameras increased. By contrast, indoor TIR measurements on the $1 \mathrm{~m}^{2}$ thermal test card showed a linear relationship with shooting distance, but thermal metrics were also under-estimated at increasing distances (red squares in Fig. 2). Moreover, global radiation levels influenced the magnitude of this error: for instance at $40 \mathrm{~m}$, mean temperatures were under-estimated by about $3.3 \mathrm{~K}$ and $1.5 \mathrm{~K}$ at radiation levels of $915 \pm 20 \mathrm{~W} / \mathrm{m}^{2}$ and $65 \pm 5 \mathrm{~W} / \mathrm{m}^{2}$, respectively (Fig. 2 A). In other words, the surface temperature of solar-heated objects was underestimated more than relatively less heated surfaces at the same distance. A similar pattern was found with $\Delta$ SD (Fig. 2 B). By contrast, $\Delta$ aggregation increased with distance (Fig. 2 D).

\subsection{Effect of surface structural complexity}

Overall, we found no effect of the surface structural complexity on the relationship between thermal metrics and shooting distance. The same decreasing pattern with increasing distance was found for the three structurally different surfaces (thermal test card surface, green wall vegetation surface and wood edge surface) and for $\Delta T_{\text {mean }}, \Delta \mathrm{SD}, \Delta$ Patch richness (and a similar increasing pattern for $\Delta$ Aggregation). However, under similar solar radiation, surfaces had different TIR responses. The thermal heterogeneity of the wood edge surface, the more structurally complex, was less under-estimated with increasing distance than the green wall and the thermal test card surfaces (Fig. $3 \mathrm{~A}$ and B).

\subsection{Surface temperature excess distributions vs. distance}

Overall, temperature excess distributions shifted down to lower values with increasing distance (Fig. 4). Under similar radiation levels, this shift was larger for the thermal test card (up to $-3 \mathrm{~K}$; Fig. 4 B) than for the green wall and the wood edge surfaces (Fig. 4 $C$, D, respectively). The range of excess temperature of the distribution curves - i.e. the spatial variation of temperature - decreased with increasing distances, from $7 \mathrm{~K}$ at $5-2 \mathrm{~K}$ at $60 \mathrm{~m}$ for the $1 \mathrm{~m}^{2}$ thermal test card in the green wall environment (Fig. 4 B). This decrease was larger for the $1 \mathrm{~m}^{2}$ thermal test card than for the green wall and the wood edge surfaces under similar solar radiation (Fig. 4 B-D). As a consequence, the maximum frequency increased with increasing distance between the surface and the TIR camera. The maximum frequency at $5 \mathrm{~m}$ for the thermal test card outdoor reached 0.18 while it increased up to 0.90 at $60 \mathrm{~m}$ (Fig. 4 B). Therefore, increasing distances caused both an under-estimation of the extreme temperature and a spatial homogenization of temperatures. We also found that shooting distance significantly modified the surface temperature distribution in the outdoor environments (ANOVAs in Table 1). Leaf temperature distributions, taken at a distance of $0.3 \mathrm{~m}$ from the surface (green curves in Fig. 4 C, D) showed larger temperature range and lower density maximum than the entire vegetation background in the green wall and wood edge environments. Note that shooting distance had no
Table 1

Results of ANOVA for the effects of shooting distance, radiation level and their interaction on the density distribution of the surface temperature excess used in Fig. 4. Temperature distributions were obtained from TIR images taken with the mobile TIR camera at various distances for the $1-\mathrm{m}^{2}$ thermal test card in the laboratory and in the green wall environments (A. and B. respectively), of the whole surface of the green wall (C.) and of the whole surface of the wood edge (D.). Values in bold indicates significance $(P<0.05)$.

\begin{tabular}{lllll}
\hline Parameter & F value & \multicolumn{3}{c}{$\boldsymbol{P}$ value } \\
\hline Distance & ${ }^{\mathrm{A}} 0.761$ & ${ }^{\mathrm{B}} 49.510$ & ${ }^{\mathrm{A}} 0.383$ & ${ }^{\mathrm{B}}<\mathbf{0 . 0 0 1}$ \\
& ${ }^{\mathrm{C}} 31.742$ & ${ }^{\mathrm{D}} 16.843$ & ${ }^{\mathrm{C}}<\mathbf{0 . 0 0 5}$ & ${ }^{\mathrm{D}}<\mathbf{0 . 0 1}$ \\
Radiation & ${ }^{\mathrm{A}} 0.079$ & ${ }^{\mathrm{B}} 34.372$ & ${ }^{\mathrm{A}} 0.778$ & ${ }^{\mathrm{B}} \mathbf{0 . 0 4 7}$ \\
& ${ }^{\mathrm{C}} 0.317$ & ${ }^{\mathrm{D}} 0.116$ & ${ }^{\mathrm{C}} 0.574$ & ${ }^{\mathrm{D}} 0.683$ \\
Dist $\times$ rad & ${ }^{\mathrm{A}} 0.039$ & ${ }^{\mathrm{B}} 1.119$ & ${ }^{\mathrm{A}} 0.844$ & ${ }^{\mathrm{B}} 0.29$ \\
& ${ }^{\mathrm{C}} 2.108$ & ${ }^{\mathrm{D}} 1.331$ & ${ }^{\mathrm{C}} 0.147$ & ${ }^{\mathrm{D}} 0.21$ \\
\hline
\end{tabular}

significant effect on the temperature distributions for the $1 \mathrm{~m}^{2}$ thermal test card in the indoor laboratory environment (ANOVA in Table $1, F_{A}=0.761, P_{A}=0.383$ ). Nevertheless, they shifted downward up to $-1 \mathrm{~K}$ with increasing distance, which is less than for outdoor surfaces (Fig. $4 \mathrm{~A}$ ).

\section{Discussion}

TIR imagery is widely used to record object/organism surface temperatures and quantify their spatial heterogeneities in ecological studies. However, some key parameters in thermography strongly impact TIR outputs. In the present study, we show that distance between the TIR camera and the object affected thermal metrics commonly used for quantifying thermal heterogeneity of surfaces. Overall, we found that shooting distance strongly modified temperature measured by the TIR camera. The relationship found between distance and mean temperature, standard deviation and patch richness for outdoor environments was non-linear, indicating a strong effect within the first $20 \mathrm{~m}$ and only a slight decrease between 20 and $80 \mathrm{~m}$. As a result, average surface temperatures were underestimated when increasing shooting distance. Interestingly, increasing shooting distance homogenised thermal mosaics with a much stronger bias in the warmer parts of the distributions. To our knowledge, this effect of shooting distance has never been quantified before. This quantification is critical for future studies that aim to assess the thermal heterogeneity available for animals and plants. Below, we explain this effect of shooting distance by the lower atmosphere composition, the size of pixels, and the influence of global solar radiation on structurally complex surfaces.

\subsection{Atmospheric composition effect}

The underestimation of mean temperature, standard deviation and patch richness might occur because of the composition of ambient atmosphere. Indeed, absorption of infrared radiation (emitted by objects) occurs due to gases and particles present in the lower atmosphere between the object and the TIR camera (Minkina and Dudzik, 2009; Kuenzer and Dech, 2013). For instance, air humidity, fog, snow, and dust can significantly distort the TIR readings (Minkina and Dudzik, 2009). This effect of atmospheric composition is suggested by the negative linear relationship between thermal metrics and the distance in the indoor environment, wherein abiotic parameters such as air temperature and humidity were more homogeneous in space and in time (see red squares at $65 \mathrm{~W} / \mathrm{m}^{2}$ in Fig. 2). Indeed, the temperature surface distributions of the TIR images for the thermal test card in the laboratory environment shifted downward by no more than $1 \mathrm{~K}$ from 1 to $50 \mathrm{~m}$, and both the maximum frequency and the 
temperature range did not change with distance in this stable environment (Fig. 4 A). By contrast, the lower atmosphere composition in the outdoor environments was probably heterogeneous along the transects. For example, the camera may have received more infrared radiation coming from nearby surfaces at close than at moderate and long distances (boundary layer properties, Oke, 1987). This effect can explain the non linear decrease of thermal metrics in outdoor transects (Fig. 4 B). Consequently, depending on the composition of the lower atmosphere during TIR measurements, a critical distance could be defined: in our case, small variations in distance induced high misestimates of surface temperature before $20 \mathrm{~m}$ while beyond this critical distance small variations in distance lead to small differences in the temperature readings. Identifying this critical distance is of key importance to reduce inaccuracies due to distance between object and TIR cameras. Moreover, concurrently with other studies (Clark, 1976; Minkina and Dudzik, 2009; Vollmer and Möllmann, 2010; Jones, 2013), we found that global radiation level altered TIR outputs and therefore modified the relationship between shooting distance and thermal metrics. Indeed, global radiation heat up the small portions of the surface that are perpendicular to the sun position, while the portions at a lower angle to the sun remain close to ambient air temperature, increasing thereby the spatial heterogeneity of surface temperatures. This effect probably amplifies the pixel size effect (see below), leading to an even larger under-estimation of thermal metrics.

\subsection{Pixel size effect}

TIR cameras are equipped with a sized sensor that provides a fixed number of pixels for any shooting distance. Therefore, the pixel size relies upon shooting distance (Online Resource 1): the further you shoot, the bigger is the pixel. This change in pixel size with distance inevitably induces modifications of the thermal information recorded by the TIR camera. Indeed, the physical borders between an object, or a thermal patch, and its surrounding may be included in the same single pixel depending on shooting distance, and in this case the pixel simply integrates the TIR information coming from both elements - i.e. a combination of subpixel temperatures (Murphy et al., 2014). The integration of subpixel temperatures likely reduces the level of heterogeneity in the TIR images. This effect is well illustrated by the response of the aggregation index to shooting distance: thermal patches became more aggregated as shooting distance increased (Fig. 4). The aggregation index relies on the number of pixels composing the landscape (McGarigal and Marks, 1994; He et al., 2000). Indeed, the number of pixels composing a $1 \mathrm{~m}^{2}$ surface area decreases with distance, causing thereby an 'apparent' increase in aggregation. The pixel size effect may also help to explain the critical distance $(20 \mathrm{~m})$ at which the rate of decrease in thermal metrics changes. The pixel size effect likely interacts with the arrangement of thermal patches. Smaller hot patches that are more spread over the surface are likely to be buffered quickly as distance increases compared to a surface composed of few large hot patches. Once the hottest patches are buffered and agglomerated with the other patches, the thermal metrics are less influenced by distance. More research is needed to test this hypothesis.

\subsection{Effect of surface structural complexity}

The relationship between shooting distance and thermal metrics was only weakly influenced by the structural complexity of surfaces (thermal test card, green wall, and wood edge). This is a quite unexpected result as the interaction between a high level of radiation and roughness of the surface is known to generate a highly diverse mosaic of temperature patches according to simple geometrical rules (Oke, 1987). We therefore expected a high spatial heterogeneity in surface temperature for the wood edge because of its three dimensional structure. However, the background of the wood edge corresponded to a deep, shaded part of the wood, which may homogenize the TIR image. Indeed, under identical weather conditions (including solar radiation) the three structurally different surfaces showed different thermal metric responses (Online Resource 8), i.e. a lower thermal heterogeneity for the wood edge surfaces than for the green wall surfaces. We also acknowledge that by starting at a $\Delta$ distance of $7 \mathrm{~m}$ in the wood edge environment, we may have missed much of the thermal differences that occur in the first metres. On the contrary, the thermal test card surface, although less structurally complex, showed a higher heterogeneity in recorded temperatures than for the two other surfaces under identical abiotic conditions. The thermal test card emits TIR radiation directly as function of incoming energy, while in the case of the green wall and the wood edge environments, the eco-physiology of plant leaves managed radiation loads and modulated their surface temperatures by evapotranspiration process (Jones, 2013). Therefore, at local scale the structural composition alone is not sufficient to infer the heterogeneity of surface temperature.

\subsection{Guidelines for the use of thermography regarding shooting distance}

We present some major guidelines to minimize inaccuracies due to distance between studied object and TIR cameras. Firstly, to yield accurate TIR measurements, emissivity of the object should be fixed in the settings of the camera according to emissivity tables (Clark, 1976). Indeed, different values of emissivity may modify the temperature readings of an object by various degrees Celsius. Therefore emissivity should be appropriately fixed for each object in the TIR image (Faye et al., 2015). Notwithstanding, parts of a same object can have different emissivity values depending on their structural composition (Rubio et al., 1997). Additionally, global solar radiation must be recorded while shooting to proceed within similar irradiance conditions. When relevant, TIR shots should be taken at low solar irradiance or during night to avoid underestimations of surface temperatures. Furthermore, to minimize the sub-pixel temperature combination onto the physical borders of the studied surface, we would recommend to manually remove the surface boundary edge - i.e. the boundary pixels - in the TIR image. However, this precaution will not exclude the inaccuracies due to sub-pixel temperature combination onto the thermal patches.

Secondly, the relationship between shooting distance and accuracy of the TIR images must be considered for data analysis. TIR studies should anticipate the influence of lower atmosphere composition (especially when outdoor) and of shooting distancerelated pixel size. Thus, we recommend reducing the shooting distance at the lowest possible distance (when feasible) to yield more accurate absolute surface temperatures. If not, atmospheric radiative transfer models could be used to correct the surface temperatures depending on atmospheric composition. For instance, MODTRAN ${ }^{\mathrm{E}} 6$ (MODerate resolution atmospheric TRANsmission) solves the radiative transfer equation including the effects of molecular and particulate absorption/emission of the atmosphere present between the thermal sensor and the studied object (Berk et al., 2014).

Object or organism body size is also a key parameter that restrains the use of thermography and the determination of shooting distance. Indeed, surface temperatures significantly affect the performance of small living organisms mainly (e.g. insects and rocky shore crustaceans, when the heat budget is driven mainly by conduction), while the thermal budget of bigger animals is more 
influenced by property of air (convective heat loss). In particular, solar radiation warm up the surface of animal's body, increasing thereby the deviation between internal and skin temperatures. However, these effects are expected to remain minor for small, dry-skin ectotherms with low thermal inertia such as most arthropods, and plant surfaces. Nevertheless, TIR shooting distance should be selected depending on the size of the organism to maximize the number of pixels covering the object. For example, at a distance of $20 \mathrm{~m}$, the pixel size was about $2 \mathrm{~cm}^{2}$ with our best TIR camera (Online Resource 1). The opportunities for behavioural thermoregulation can therefore only be assessed at $20 \mathrm{~m}$ and below for organisms with body size $>2 \mathrm{~cm}$, assuming that the organism itself integrates surface temperatures throughout its whole body (Woods et al., 2016).

\section{Conclusion}

In conclusion, our study reveals that distance between the object and the TIR camera is a major modifier of measured thermal heterogeneity. Shooting distance causes errors and underestimates surface temperatures. Researchers should therefore select the shooting distance according to a trade-off between body size, TIR camera specifications (especially field of view), the hypothetical surface temperature (if the object surface temperature is heated), and the absolute level of accuracy required. These recommendations apply for any field of research where thermography is used.

\section{Acknowledgements}

We are grateful to Christelle Breion (www.musee-balzac.fr) for allowing us to work in the Château de Saché, and Sophie CauvyFraunie for her help in the fieldwork. Thanks to Damien Legaie for clarifying comments on the manuscript. This work was partly conducted within the project Microclimite "From global to microclimate change" (ANR-10-BLAN-1706-02) and the project "Adaptive management in insect pest control in thermally heterogeneous agricultural landscapes" (ANR-12-JSV7-0013-01) both funded by the Agence Nationale pour la Recherche (ANR, www. agence-nationale-recherche.fr).

\section{Appendix A. Supplementary material}

Supplementary data associated with this article can be found in the online version at http://dx.doi.org/10.1016/j.jtherbio.2015.11. 011.

\section{References}

Bellvert, J., Zarco-Tejada, P.J., Girona, J., Fereres, E., 2013. Mapping crop water stress index in a 'Pinot-noir'vineyard: comparing ground measurements with thermal remote sensing imagery from an unmanned aerial vehicule. Precis. Agric. 1-16.

Berk, A., Conforti, P., Kennett, R., Perkins, T., Hawes, F., van den Bosch, J. 2014. MODTRAN6: a major upgrade of the MODTRAN radiative transfer code. In: Proceedings of the SPIE Defense + Security, International Society for Optics and Photonics, pp. 90880H-90880H.

Caillon, R., Suppo, C., Casas, J., Woods, H., Pincebourde, S., 2014. Warming decreases thermal heterogeneity of leaf surfaces: implications for behavioural thermoregulation by arthropods. Funct. Ecol. . http://dx.doi.org/10.1111/ 1365-2435.12288

Cilulko, J., Janiszewski, P., Bogdaszewski, M., Szczygielska, E., 2013. Infrared therma imaging in studies of wild animals. Eur. J. Wildl. Res. 59 (1), 17-23.

Clark, J.A., 1976. Effects of surface emissivity and viewing angle on errors in thermography. Acta Therm. 1, 138-141.

Croon, G.W., McCullough, D.R., Olson, C.E., Queal, L.M., 1968. Infrared scanning techniques for big game censusing. J. Wildl. Manag. 32, 751-759.

Faye, E., Rebaudo, F., Yánez, D., Cauvy-Fraunié, S., Dangles, O., 2015. A toolbox for studying thermal heterogeneity across spatial scales: from unmanned aerial vehicle imagery to landscape metrics. Methods Ecol. Evol. . http://dx.doi.org/ 10.1111/2041-210X.12488

Gates, D.M., 1980. Biophysical ecology. Dover Publications, New York, USA.

He, H.S., DeZonia, B.E., Mladenoff, D.J., 2000. An aggregation index (AI) to quantify spatial patterns of landscapes. Land. Ecol. 15 (7), 591-601.

Hill, R.W., Christian, D.P., Veghte, J.H., 1980. Jackrabbit ears: surface temperatures and vascular responses. Science 194, 436-438.

Inagaki, M.N., Nachit, M.M., 2008. Visual monitoring of water deficit stress using infra-red thermography in wheat. In: Appels, Rudi, Eastwood, Russell, Lagudah, Evans, Langridge, Peter, Mackay, Michael, McIntyre, Lynne (Eds.), Proceedings of the 11th International Wheat Genetics Symposium, Sydney University Press.

Jackson, R.D., Idso, S.B., Reginato, R.J., Pinter, P.J., 1981. Canopy temperature as a crop water stress indicator. Water Resour. Res. 17 (4), 1133-1138.

Jones, H.G., 2013. Plants and Microclimate: A Quantitative Approach to Environmental Plant Physiology, third edition. Cambridge University Press, Cambridge.

Jones, H.G., Stoll, M., Santos, T., De Sausa, C., Chaves, M.M., Grant, O.M., 2002. Use of infrared thermography for monitoring stomatal closure in the field: application to grapevine. J. Exp. Bot. 53 (378), 2249-2260.

Kerr, J.T., Ostrovsky, M., 2003. From space to species: ecological applications for remote sensing. Trends Ecol. Evol. 18 (6), 299-305.

Kingsolver, J.G., 2009. The Well-temperatured biologist. Am. Nat. 174 (6), 755-768. Kuenzer, C., Dech, S. 2013. Thermal Infrared Remote Sensing. Springer, London, UK.

Lathlean, J., Seuront, L., 2014. Infrared thermography in marine ecology: methods, previous applications and future challenges. Mar. Ecol. Prog. Ser. 514, 263-277.

Koerner, C., Basler, D., 2010. Phenology under global warming. Science 327 (5972), 1461-1462.

McCafferty, D.J., Gilbert, C., Thierry, A.M., Currie, J., Le Maho, Y., Ancel, A., 2013. Emperor penguin body surfaces cool below air temperature. Biol. Lett. 9 (3) 20121192.

McGarigal, K., Marks, B.J., 1994. Fragstats: Spatial Pattern Analysis Program for Quantifying Landscape Structure. Oregon State University, Forest Science Department, Portland, OR.

Meron, M., Tsipris, J., Orlov, V., Alchanatis, V., Cohen, Y., 2010. Crop water stress mapping for site-specific irrigation by thermal imagery and artificial reference surfaces. Precis. Agri. 11 (2), 148-162.

Minkina, W., Dudzik, S., 2009. Uncertainties of measurements in infrared thermography, Infrared Thermography: Errors and Uncertainties. John Wiley and Sons, Ltd., Chichester, UK.http://dx.doi.org/10.1002/9780470682234.ch5.

Murphy, S.W., Oppenheimer, C., de Souza-Filho, C.R., 2014. Calculating radiant flux from thermally mixed pixels using a spectral library. Remote Sens. Environ. 142, 83-94.

Nienaber, J., Thomton, J., Horning, M., Polasek, L., Mellish, J.A., 2010. Surface temperature patterns in seals and sea lions: a validation of temporal and spatial consistency. J. Therm. Biol. 35 (8), 435-440.

Norman, J.M., Becker, F., 1995. Terminology in thermal infrared remote sensing of natural surfaces. Remote Sens. Rev. 12 (3-4), 159-173.

Oke, T.R., 1987. Boundary Layer Climates. Vol. 5. Routledge, Taylor and Francis Group, New York.

Ørtisland, N.A., 1968. Variations in the body surface temperature of the harp seal. Acta Physiol. Scand. 73, 35-36.

Pincebourde, S., Woods, H.A., 2012. Climate uncertainty on leaf surfaces: the biophysics of leaf microclimates and their consequences for leaf-dwelling organisms. Funct. Ecol. 26 (4), 844-853.

Pincebourde, S., Sanford, E., Casas, J., Helmuth, B., 2012. Temporal coincidence of environmental stress events modulates predation rates. Ecol. Lett. 15 (7) 680-688.

Pincebourde, S., Sanford, E., Helmuth, B., 2013. Survival and arm abscission are linked to regional heterothermy in an intertidal sea star. J. Exp. Biol. 216 (12), 2183-2191.

Quattrochi, D.A., Luvall, J.C., 1999. Thermal infrared remote sensing for analysis of landscape ecological processes: methods and applications. Landsc. Ecol. 14 (6), 577-598.

R Development Core Team, 2015. R: A Language and Environment for Statistical Computing. R Foundation for Statistical Computing, Vienna, Austria.

Rubio, E., Caselles, V., Badenas, C., 1997. Emissivity measurements of several soils and vegetation types in the 8-14 mm wave band: analysis of two field methods. Remote Sens. Environ. 59 (3), 490-521.

Scherrer, D., Koerner, C., 2010. Infra-red thermometry of alpine landscapes challenges climatic warming projections. Glob. Chang. Biol. 16 (9), 2602-2613.

Tattersall, G.J., Cadena, V., 2010. Insights into animal temperature adaptations revealed through thermal imaging. Imag. Sci. J. 58 (5), 261-268.

Tonolla, D., Acuña, V., Uehlinger, U., Frank, T., Tockner, K., 2010. Thermal heterogeneity in river floodplains. Ecosystems 13 (5), 727-740.

Vollmer, M., Möllmann, K.P., 2010. Infrared Thermal Imaging: Fundamentals, Research and Applications. John Wiley and Sons, New York.

Woods, H.A., 2013. Ontogenetic changes in the body temperature of an insect herbivore. Funct. Ecol. 27 (6), 1322-1331.

Woods, H.A., Dillon, E.M., Pincebourde, S., 2016. The roles of microclimatic diversity and of behaviour in mediating the responses of ectotherms to climate change. J. Therm. Biol. http://dxdoi.org/10.1016/j.jtherbio.2014.10.002. 